\title{
Articulación en la Promoción Ambiental desde la Tríada: Escuela, Familia, Comunidad
}

Autora: Adriana Del Rosario Mejías Vetancourt Universidad Pedagógica Experimental Libertador, UPEL adrimejivetancourt@hotmail.com

Barinas, Venezuela

\section{Resumen}

El presente trabajo de investigación tiene como objetivo principal, generar una aproximación teórica de la Articulación en la Promoción Ambiental desde la Tríada: Escuela, Familia, Comunidad en la Escuela Básica "Cinqueña III", municipio Barinas del estado Barinas. La naturaleza de esta investigación es cualitativa apoyada en el enfoque fenomenológico del paradigma socio crítico. Los informantes claves se consideraron cinco (05), los cuales, fueron elegidos a criterio de la investigadora, según los actores que se consideran vinculantes: un directivo, un docente, un representante, un miembro del consejo educativo, un miembro del consejo comunal. Entre las técnicas de recolección de la información se tienen: la observación participante y la entrevista cualitativa a profundidad. En cuanto a las técnicas para el análisis de la información se contempla la categorización, codificación y triangulación, acompañada de la fase descriptiva e interpretativa. Seguidamente, se efectuará una matriz comparativa para el análisis de la información recolectada y se procederá a determinar los hallazgos encontrados como resultado del abordaje del tema de investigación en la Escuela Básica Cinqueña III, municipio Barinas del estado Barinas.

Palabras clave: articulación; promoción ambiental; escuela; familia; comunidad.

Fecha de Recepción: 25-06-2016 Fecha de Aceptación: 29-07-2016 


\title{
Joint en Environmental Promotion from the Triad: School, Family, Community
}

\begin{abstract}
The present research has as main objective to generate a theoretical approach of the Joint Environmental Promotion from the Triad: School, Family, Community Primary School "Cinqueña III" town Barinas state of Barinas. The nature of this research is supported by the phenomenological qualitative approach critical partner paradigm. Key informants were considered five (05), which were chosen at the discretion of the investigator, according to the actors who are considered binding: a manager, a teacher, a representative, a member of the school board, a member of the community council. Among the techniques of information collection are: participant observation and qualitative depth interview. As techniques for analyzing information categorization, coding and triangulation, accompanied by descriptive and interpretative phase it is contemplated. Then, a comparative matrix is made to analyze the information collected and shall determine the findings as a result of addressing the issue of research in the Basic School Cinqueña III, municipality of Barinas Barinas state.
\end{abstract}

Keywords: joint environmental promotion; school; family; community. 


\section{Introducción}

Las sociedades avanzan a un ritmo acelerado en cuanto a tecnología se refiere, aportando aspectos positivos para el desarrollo de las naciones. Sin embargo, estos avances ocasionan daños irreversibles a la colectividad en general como lo es el deterioro al ambiente. Las condiciones que brinda el escenario mundial en estos momentos en materia ambiental exigen plantear una Educación Ambiental crítica encargada de estudiar las realidades acontecidas en el planeta y sus consecuencias. Es por ello, que le compete al sistema educativo, el rescate de actividades y valores que vayan en beneficio de la preservación del ambiente a fin de crear conciencia individual y colectiva, de la necesidad de proteger los recursos esenciales para la vida humana.

Cabe agregar, que la formación ambiental es obligación de todos y debe basarse en la integralidad social, con el propósito de aprender dichos conocimientos y actuar en forma razonable hasta experimentar transformaciones personales y sociales de identidad con su entorno.

En ese orden de ideas, se presenta la investigación titulada: Articulación en la Promoción Ambiental desde la Tríada: Escuela, Familia, Comunidad. (Caso de estudio: Escuela Básica "Cinqueña III", municipio Barinas del estado Barinas), la cual, se encuentra concebida dentro de la naturaleza cualitativa, bajo un enfoque fenomenológico y comprende la siguiente estructura:

Capítulo I: describe la Realidad del Contexto, ontología del hecho, propósito general, propósitos específicos y la razón del estudio.

Capítulo II: se presenta las implicaciones epistemológicas que abarcan los antecedentes históricos e investigativos, la aproximación teórica y definición de términos.

Capítulo III: presenta los referentes paradigmáticos que incluyen la naturaleza de la investigación, postura metodológica, informantes claves, técnicas de recolección de la información y las técnicas para el análisis de la información. Asimismo, se anexan las referencias y sus respectivos anexos. 
En lo que refiere al propósito general de la investigación es generar una aproximación teórica de la Articulación en la Promoción Ambiental desde la Tríada: Escuela, Familia, Comunidad en la Escuela Básica "Cinqueña III", municipio Barinas del estado Barinas. Y los propósitos específicos son detectar la realidad en la articulación que prevalece desde la Educación Ambiental en la triada: Familia, escuela, comunidad. Establecer la articulación desde la triada: Familia, escuela, comunidad y la Educación Ambiental. Categorizar una aproximación teórica a partir de la Articulación en la Promoción Ambiental desde la Tríada: Escuela, Familia, Comunidad.

\section{Fundamentación teórica}

\section{Aproximación Teórica}

Al realizar un trabajo de investigación, es fundamental comenzar por describir las fases que este implica para conformar su estructura, dentro de las cuales se encuentran la categorización, contrastación y teorización. En este sentido, es preciso destacar que ninguna de estas tres fases puede llevarse a cabo de forma aislada, toda vez que el cerebro no lo permite.

La razón para justificar dicha apreciación es que el poder mental de los individuos es tan complejo que deambulada en la búsqueda de hallar sentido a las cosas estableciendo conexión entre los elementos que se le presentan y es justamente de donde parte el análisis, la necesidad de justificar todo lo que sucede en el entorno y organizarlo coherentemente dando lugar a la teorización, proceso que, de acuerdo con Martínez (2006),

Utiliza todos los medios disponibles a su alcance para lograr la síntesis final de un estudio o investigación. Más concretamente, este proceso trata de integrar en un todo coherente y lógico los resultados de la investigación en curso, mejorándolo con los aportes de los autores reseñados en el marco teórico referencial después del trabajo de contrastación (pág. 10).

Esto quiere decir, que la teorización tiene por norte, engranar toda la 
información a la que pueda acceder para organizarla, sistematizarla y en consecuencia dar lugar a la culminación del estudio con aportes significativos para solventar la problemática. Ciertamente, el autor se fundamenta en la obra de Descartes quien en su oportunidad expresó que para poder conocer a profundidad una problemática, era preciso ante todo dividirla en cuantas partes le fuere posible al investigador, tomando en consideración hasta los aspectos que a simple vista parecieren insignificantes.

Ciertamente, este aspecto no representa mayor dificultad para los individuos de la especie humana en razón de su inteligencia, la cual de forma innata gira en torno de la indagación de métodos para organizar las cosas conforme a sus concurrencias o analogías, origen, caracteres e interacción. De modo que, una vez, el investigador haya reflexionado respecto a los datos obtenidos en su proceso de indagación le es posible ir construyendo de forma mental, paulatina las categorías propiedades y atributos que inciden en la problemática estudiada y por ende construir una teoría valida. Esta aseveración concuerda con lo expresado por Martínez (ob.cit.), para quien:

La teoría es, por tanto, un modelo ideal, sin contenido observacional directo, que nos ofrece una estructura conceptual inteligible, sistemática y coherente para ordenar los fenómenos; de manera más concreta, suele consistir en un sistema de hipótesis, fórmulas legaliformes y hasta leyes ya establecidas, de modo que su síntesis puede incluir desde lo plenamente conocido hasta lo meramente sospechado (pág. 12).

A partir de allí es posible afirmar que teorizar en los trabajos de investigación cualitativa, como es el caso del presente estudio, involucra dar luz a las ideas del investigador, intuirlas, crear una representación sensata, organizada e interpretativa, exigiendo la exploración plena y valoración de las diversas perspectivas que surjan del análisis de la problemática que ocupa a quien indaga. 


\section{Postura Metodológica}

El enfoque metodológico que se empleará en el estudio fenomenológico. Para Husserl citado por Rusque (2007), "la experiencia del fenomenólogo es el fenómeno. El fenomenólogo no busca el ser detrás del fenómeno, sino su esencia" (pág. 22). De ahí, el interés de la investigadora se centre en los hechos existentes de la individualidad de los sujetos, y en el modo irreductible de su aparición. Lo que hace deducir que para la fenomenología el instrumento de conocimiento es la intuición.

En correspondencia con lo descrito, Pocovi (2004), señala que "el punto de partida de la fenomenología es la vida de las personas enfocándose en como las experiencias, significados $y$ emociones son concebidos $y$ aprendidos" (pág. 77). Asimismo, se asumirá que la aplicación de este método, es sin supuestos, no especulativo, se describirá el fenómeno tal como es experimentado, empírico, científico como un método y generalizable a través de las situaciones. En cuanto a las etapas a cumplirse se atenderá a lo planteado por Martínez (2006), el cual considera:

Etapa Previa: En la misma se seleccionarán los actores sociales, las técnicas de recolección de datos, técnicas de análisis de la información, documentación teórica (referencias y estudios previos).

Etapa descriptiva: constituye en el análisis de las entrevistas y observaciones a fin de destacar los indicadores más importantes que explican el estudio, así como la comparación entre las diversas opiniones, para contrastar la realidad latente.

Etapa Estructural: se enfocará en la interpretación de las categorías, para lo cual se hará uso de la técnica de la reducción, la cual, permitirá seleccionar los aspectos más relevantes de los informantes, estableciendo las semejanzas y diferencias entre los mismos.

Discusión de Resultados: una vez procesada parte de la información, se procederá a la aplicación de triangulaciones, que determinarán las 
coincidencias de la problemática en estudio, para poder emitir juicios de valor en relación al estudio, estos son, interpretaciones, relaciones, análisis, teorización, entre otros. Asimismo, fundamentará la elaboración aportes finales del trabajo investigativo.

\section{Informantes Claves}

Uno de los recursos imprescindibles en la investigación cualitativa son los informantes claves, definido por Robledo (2009), como:

Aquellas personas que por sus vivencias, capacidad de empatizar y relaciones que tienen en el campo pueden apadrinar al investigador convirtiéndose en una fuente importante de información a la vez que le va abriendo el acceso a otras personas y a nuevos escenarios (pág. 12).

\section{Técnicas de Recolección de la Información}

En la investigación fenomenológica las técnicas de recolección de información deben permitir que la investigadora se sumerja en el fenómeno de estudio, por lo tanto tienen que ser flexibles y abiertas. En este caso, se utilizarán: la observación participante y la entrevista cualitativa a profundidad. Sin obviar cualquier otra que pudiera adaptarse a la naturaleza de la investigación y sea beneficiosa para la misma. Para Robledo (ob.cit.), la observación participante:

Es una técnica que implica la interacción entre el investigador y los grupos sociales. Su objetivo es recoger datos de modo sistemático directamente de los contextos y se fundamenta en el principio de la convivencia personal del investigador con el grupo o institución que se investiga (pág. 134).

Para llevar a la práctica esta técnica se contemplarán tres momentos: un primer momento denominado acceso al escenario, en el cual, la investigadora debe buscar la aceptación del grupo; un segundo momento: estancia en el escenario, donde la investigadora involucrada con el grupo 
realizará el proceso de observación interactiva y un tercer momento: retirada del escenario en el cual la investigadora se retirará del grupo en forma amigable.

Por otra parte, Robledo (ob.cit.), refiere la entrevista a profundidad como "una de las técnicas más usadas junto con la observación participante y señala que la misma busca encontrar lo más importante y significativo para los informantes sobre los acontecimientos y dimensiones subjetivas" (pág. 69). Lo que se traduce a un encuentro cara a cara, no estructurado, donde se empleará una conversación; orientada por un guía de entrevista. Durante su ejecución, también será posible grabar el testimonio de los informantes.

\section{Técnicas para el análisis de la información}

En atención a la naturaleza de la investigación las estrategias utilizadas en el análisis de la información en el estudio titulado: Articulación en la Promoción Ambiental desde la Tríada: Escuela, Familia, Comunidad en la Escuela Básica "Cinqueña III", municipio Barinas del estado Barinas, serán la categorización, codificación y triangulación, que a continuación se detallan:

Categorización: Según Martínez (2006), consiste en estructurar una imagen representativa, como patrón lógico, de la configuración del fenómeno en estudio; dándole sentido a todos los componentes, este proceso se llevará a cabo a través de procesos de organización e interpretación de los datos cualitativos. De aquí surgirá el sistema de categorías.

Codificación: La codificación corresponde a una transformación efectuada según reglas precisas de los datos brutos del texto. Es la transformación que por descomposición, agregación y enumeración permitirá desembocar en una representación del contenido, o de su expresión, susceptible de ilustrar al analista sobre las características del texto que servirán de índices. Asimismo, en la interpretación de los resultados se utilizará la triangulación, la cual, según Louis citado por Robledo (2009), la triangulación consiste en: 
...la verificación de que una interpretación es acertada porque al ser considerada de manera repetida hay coincidencia y no divergencia en la conclusión que se deriva de ella (...). Así, debe haber similitud en lo interpretado de los eventos producto de entrevista, observación, análisis de documentos y registros, o entre lo propuesto por más de dos especialistas. El término no tiene relación exacta con el número de expertos ni de técnicas involucradas, sino más bien con el carácter iterativo del proceso (pág. 44).

De ahí que la triangulación, en este estudio, permitirá contrastar reiterativamente la evidencia que será analizada y que se recopilará desde visiones y momentos diferentes, esto es, entrevista preliminar, observación participante y entrevista a profundidad. Ahora bien, la triangulación llevará de modo explícito a validar la evidencia, para lo cual, el procedimiento a ejecutar será:

La Fase Descriptiva, consiste en, registrar la evidencia recopilada mediante la triangulación. Ésta tendrá como fin, informar al lector, de la manera más objetiva posible la situación que está siendo objeto de estudio. En otras palabras, revelará en primer lugar, lo que la investigadora ve o percibe, a través de la observación participante. $\mathrm{Y}$, en segundo lugar, plasmará las apreciaciones que el práctico (Escuela-Familia-Comunidad), da sobre sus actuaciones cotidianas.

La Fase de Categorización, emergerá de los registros sobre la evidencia, pues, no es más que su clasificación. Hecho que llevará a situarla, en un conjunto de patrones y tendencias que serán identificados subjetivamente por la investigadora. Es decir, la categorización será la fase que llevará a ordenar la evidencia relevante para el estudio, a partir de las percepciones de la investigadora y la experiencia contada por los mismos actores.

La Fase Interpretación y Explicación permite integrar los patrones y tendencias (productos de la fase anterior), a fin de realizar las interpretación, 
explicaciones y relaciones con un sentido crítico y objetivo sobre la información obtenida.

\section{Conclusiones}

Las principales conclusiones que se derivan del trabajo de investigación son las siguientes:

La Educación Ambiental es comprendida por los tres sujetos de estudios, como aquella que se encarga de orientar a las personas a prácticas sociales-conservacionistas, en pro de promover actividades amigables o que coadyuven a la conservación y preservación del medio ambiente. Así mismo, los sujetos reconocen la importancia de los entes educativos, como espacios reflexivos para cambiar las actitudes de las personas, lo que se persigue es un nuevo sujeto social más responsable hacia la naturaleza con carácter vinculante entre la escuela-comunidad.

Las personas entrevistadas se identificaron con la Educación y Gestión Ambiental como activas, con alta responsabilidad con el medio ambiente. Sin embargo, ellos señalaron que hay que cambiar los estilos de vida de las personas (no tanto consumismo, no tanto materialismo), sino un estilo de vida que permita el disfrute de los beneficios de la naturaleza de una manera sustentable y sostenible. Para lograr lo anteriormente señalado, ven de forma acertada la inclusión de la Educación Ambiental en el curriculum educativo, realizar jornadas comunicativas audio-visuales. Además, se observó que la implementación de las brigadas escolares son una estrategia acertada, porque permiten una mayor difusión de la información ambiental tanto para la escuela como para la comunidad.

Es importante resaltar, en la categoría Educación y Gestión Ambiental los informantes señalaron la necesidad de realizar investigaciones en los problemas medioambientales, lo cual evidencia la necesidad de realizar un diagnóstico para exponer las diferentes alternativas de solución. 
La triada escuela-familia-comunidad bajo enfoque de Educación Ambiental, se constató que sí existe. Sin embargo, los sujetos de estudios señalaron la necesidad de promover valores familiares y educativos en los estudiantes en formación, en aras de cambiar su razonamiento frente a la problemática ambiental que se presente en la institución educativa o en la comunidad. Así mismo, los informantes manifestaron que las reuniones de padres y representantes son un escenario para impulsar la educación ambiental. Por otro lado, no se descartan otras actividades educativasambientales como por ejemplo: afiches, murales, carteleras informativas, campañas con perifoneo, entre otras.

Los sujetos de estudios coinciden en que una Educación Ambiental permite visualizar las problemáticas ambientales de la escuela o comunidad, ofrecer a su vez posibles soluciones con todos los actores sociales: familiaescuela-comunidad. En ese sentido, la preservación y conservación del medio ambiente afecta de manera positiva la calidad de los individuos de un sector 0 región.

\section{Referencias}

Martínez, M. (2006). Validez y Confiabilidad de la Metodología Cuantitativa. Volumen 27, № 2. Revista Paradigma.

Martínez, M. (2006). La Nueva Ciencia, Su desafió, lógica y método. Editorial Trillas. México.

Pocovi, P. (2004). Dos Visiones que se complementan: La Investigación Cualitativa y el Enfoque Fenomenológico. Buenos Aires: Revista Época. № 43.

Robledo, J. (2009). Observación participante: informantes claves y rol del investigador. Centro de Investigaciones Nure, № 42. Nicaragua.

Rusque, A. (2007). De la diversidad a la unidad en la investigación cualitativa. Caracas: Vadell Hermanos Editores. 


\section{Adriana Del Rosario Mejías Vetancourt \\ e-mail: adrimejivetancourt@hotmail.com}

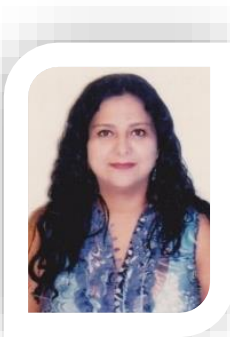

Nació en Barinas Edo. Barinas, actualmente cursando Maestría en Educación Ambiente y Desarrollo (2014) en la Universidad Pedagógica Experimental Libertador. Con Licenciatura en Educación, Mención Educación Integral (2010); de la misma Universidad. Ingresó al Ministerio del

Poder Popular para la Educación en el año 2001, como Docente de Aula, impartiendo la cátedra de Educación para la Salud de 1er año y Biología de 2do 3er y 4to año. En la actualidad se desempeña como Docente en Recursos para el Aprendizaje (Plan Lector), en la Biblioteca de la Unidad Educativa "Cinqueña III", Institución a la cual pertenece desde que inició su carrera.

El contenido de este manuscrito se difunde bajo una Licencia de Creative Commons ReconocimientoNoComercial-Compartirlgual 4.0 Internacional 